\title{
The Research of Image Feature Matching Method Based on CH-SURF
}

\author{
Zhaodi Wang \\ School of Information Engineering \\ Zhengzhou University \\ Zhengzhou ,China \\ wzdapple@126.com
}

\author{
Jicai Deng \\ School of Information Engineering \\ Zhengzhou University \\ Zhengzhou,China \\ iejcdeng@zzu.edu.cn
}

\begin{abstract}
Since SURF (Speeded-Up Robust Features) algorithm is designed for gray images in feature extraction and recognition tasks, it can't recognize some objects of similar shape but different color. CH-SURF algorithm is proposed,which combines Color Histogram feature and SURF feature together. CH-SURF algorithm firstly chooses color histogram feature of target image to reach rough localization, then in the defined small pixel region, uses SURF algorithm to achieve feature matching. Finally simulation experiments are carried out under vs2008 platform, and results show that the proposed algorithm has lower computation time than the original SURF. At the same time,it can improve the accuracy of feature matching to some extent.
\end{abstract} vs2008

Keywords-color Histogram;CH-SURF; feature matching;

\section{INTRODUCTION}

SURF[1] algorithm is the local feature point extraction algorithm.It is proposed by Herbert Bay in 2006. The characteristic of this algorithm is its invariance to rotation,scale and brightness, and the stability to angle change, affine transformation and noise. Besides,SURF algorithm adopts the theory of integral image,box filter, Haar response and Fast Hessian Detector,so it improves computation efficiency. Nowadays SURF algorithm has become prevalent in nearly several areas of computer vision,from face recognition[2], to palmprint identification[3], to remote sensing image registration[4] and Panoramic Mosaic fields.However in the feature detection and description,SURF algorithm depends on the local texture feature of image and ignores color information,so it has not enough ability to describe some image which have obvious global features.

Color feature is image global features[5], it has complementarity relation with SURF feature,in addition color feature is insensitive to image rotation, translation and scale transformation.So in this paper, we proposed to use CH-SURF algorithm of combining color features and SURF feature, and to achieve target localization. The remaining of this paper is organized as follow.Section I extracts color feature to match, and localizes target image rough position,Section II in the given region detects the interest SURF points, then by means of similarity matching method, finds target image position in the region of source image and achieves target localization task.Section III carries out some experiments to test the matching performance of $\mathrm{CH}-\mathrm{SURF}$ algorithm.

\section{FEATURE MATCHING BASED ON CH-SURF ALGORITHM}

CH-SURF algorithm is the combination of Color Histogram and SURF feature matching algorithm.It primarily extracts image color feature in the HSV space, and localizes target image rough position according to the principle of color matching.Then in the located region,extracts source image SURF points, and compare them with those points extracted from the target image.Finally by similarity matching method,completes target localization.This process includes two steps:1)color histogram matching;2) SURF feature matching.

\section{A. Color histogram matching}

This method is a widely used feature extraction and recognition method,it mainly includes two parts:

1) color histogram feature extraction

we begin by transforming the image from RGB space to HSV space. Since the H,S,V component has different sensitive to lightness respectively, they need to be quantized in different level, such as 8 level for $\mathrm{H}$ component, 3 level for $\mathrm{V}$ and $\mathrm{S}$ respectively, the quantitative formula is defined on Eq.1:

$$
\begin{aligned}
& H=\left\{\begin{array}{ll}
0, & h \in[316,20] \\
1, & h \in[21,40] \\
2, & h \in[41,75] \\
3, & h \in[76,155] \\
4, & h \in[156,190] \\
5, & h \in[191,270] \\
6, & h \in[271,295] \\
7, & h \in[296,315]
\end{array} \quad S= \begin{cases}0, & s \in[0,0.2] \\
1, & s \in[0.2,0.7]\end{cases} \right. \\
& V= \begin{cases}0, & v \in[0,0.2] \\
1, & v \in[0.2,0.7] \\
2, & v \in[0.7,1]\end{cases}
\end{aligned}
$$

According to the above formula, synthesizing three components into a one-dimensional feature vector $Q$

$$
Q=9 H+3 S+V
$$

Finally, by calculation, $8 * 3 * 3=72$ bin one-dimension color histogram can be obtained,and ready for the next image matching task.

2) color features similarity matching 
Correlation matching is the method that is used to measure the similarity of different feature, its computation formula is defined on Eq.3:

$$
d\left(H_{1}, H_{2}\right)=\frac{\sum\left(H_{1}-\overline{H_{1}}\right)\left(H_{2}-\overline{H_{2}}\right)}{\sqrt{\sum\left(H_{1}-\overline{H_{1}}\right)^{2} \sum\left(H_{2}-\overline{H_{2}}\right)^{2}}}
$$

Where $H_{1}$ represents the color histogram of target image, $\mathrm{H}_{2}$ represents the color histogram of source image, $d\left(H_{1}, H_{2}\right)$ represents they similarity. While for Eq.3,the bigger this similarity is, the better matching degree shows. So by calculation, the best matching point can be obtained when the similarity $d\left(H_{1}, H_{2}\right)$ achieves maximum,then we regards this point as the center to localize the general position of target image among the source image.

\section{B. SURF feature matching}

On the basic of the former step, we first extract the SURF feature points in both the target image and the localized source image respectively, assign these points a dominant orientation and make them generate descriptors vectors.Then combining Hessian matrix trace and Euclidean distance together, the similarity measurement of different descriptors is performed to complete the target image localization. And the realization process of SURF includes three parts:

\section{1) Detection of SURF feature points}

In order to detect SURF feature points, different scale space[6] need to be built.Given an two-dimension image $I(x, y)$ and Gaussian kernel function $G(x, y, \sigma)$, let them perform convolution operation.

$$
L(x, y, \sigma)=I(x, y) * G(x, y, \sigma)
$$

Then at every layer of scale space,the extreme value point of image is be detected by Hessian matrix.Given a point $X(x, y)$ in an image $I(x, y)$, its Hessian matrix at scale $\sigma$ is defined as:

$$
H(X, \sigma)=\left[\begin{array}{c}
L_{x x}(X, \sigma), L_{x y}(X, \sigma) \\
L_{x y}(X, \sigma), L_{y y}(X, \sigma)
\end{array}\right]
$$

Where $L_{x x}(X, \sigma)$ represents the convolution of the Gaussian second order partial derivative function with the image $I(x, y)$ at point $X(x, y)$, and similarly for $L_{x y}(X, \sigma)$ and $L_{y y}(X, \sigma)$. In order to decrease the computation burden,SURF algorithm use box filter to approximate the second order Gaussian partial derivative, and introduce the concept of the integral image. Thus, the convolution of each box filter in the $\mathrm{x}, \mathrm{y}$ and $\mathrm{xy}$ direction partial derivative function can be denoted by $D_{x x}, D_{x y}$ and $D_{y y}$ respectively,the approximated determinant of Hessian matrix can be denoted as

$$
\operatorname{det}(\text { Hessian })=D_{x x} * D_{y y}-\left(0.9 D_{x y}\right)
$$

After each scale space is calculated, a non-maximum suppression in a $3 \times 3 \times 3$ neighborhood is performed, let each point compare with its 26 neighbors to obtain these stable SURF key points.

\section{2) Description of SURF feature point}

The generation of SURF feature descriptor includes two parts: dominant orientation assignment and descriptor vector generation. Firstly, The Haar wavelet responses of pixels in both $\mathrm{x}$ and $\mathrm{y}$ direction are calculated and weighted statistical within a circular neighborhood of radius ${ }_{6}^{\sigma}$ (where ${ }^{\sigma}$ represents scale value). And then from the $\mathrm{x}$ axis,the sum of Haar wavelet responses within a circular segment covering an angle of $60^{\circ}$ origin is calculated to yield a local orientation vector ${ }^{2}$ ( Fig.1). In the same manner we can calculated once every $5^{\circ}$ in the whole circle region,so 72 different orientation vector $L_{1} 、 L_{2} \ldots \ldots L_{72}$ are got,and the longest orientation of vector is defined as the dominant orientation of this SURF key point.

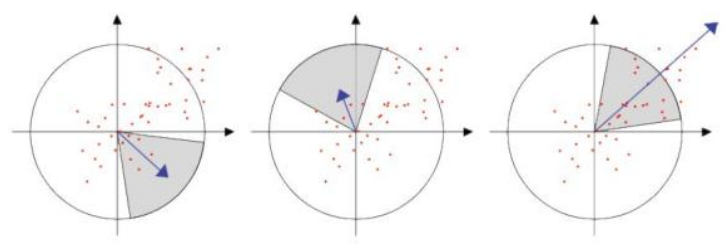

Figure 1. Orientation Assignment

Secondly, in order to generate SURF descriptor vector,we need to construct a square window with size $20 \sigma$ around the interest point,and orient its direction along dominant orientation. Next divide the descriptor window into $4 \times 4$ sub-regions( Fig.2), in which the horizontal and vertical Haar wavelet responses $d_{x}$ and $d_{y}$ are calculated and summed up, similarly for the absolute values of the responses. Thus each sub-region has four-dimensional descriptor vector $V$ constructed as Eq.7, and the length of descriptor vector is $16 \times 4=64$.

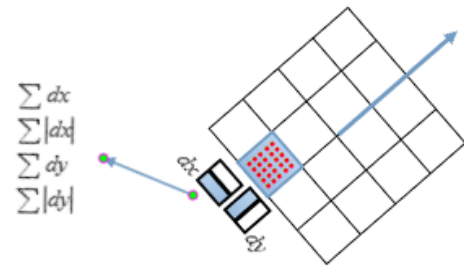

Figure 2. Descriptor Components

$$
V=\left(\sum d x, \sum d|x|, \sum d y, \sum d|y|,\right)
$$

\section{3) Matching of SURF feature}

After SURF descriptors vector generation,we use two kind of methods to measure the similarity. Firstly the method of Hessian matrix trace is implemented, because when different feature points have the same contrast intensity, their trace of Hessian matrix are the same sign; while when they have not, their trace are the contrary sign. So according to this theory, we can choose those points that satisfy this matching conditions and give up those do not.Next 
Euclidean distance(Eq.8) method is implemented among the chosen key points to match.

$$
D_{i j}=\left[\sum_{m=0}^{m-n}\left(X_{i m}-X_{j m}\right)^{2}\right]^{1 / 2}
$$

Where $X_{i m}$ represents $m$ th element of $i$ th feature descriptor in the target image; $X_{j k}$ represents $k$ th element of $j$ th feature descriptor in the source image; $n$ represents 64dimension.The Euclidean distance between all SURF descriptor vectors are computed to find minimum Euclidean distance $D_{a 1}$ and the next-minimum Euclidean distance $D_{a 2}$.Then a good match is formed only if the ratio $D_{a 1} / D_{a 2}$ is greater than 0.7 , Otherwise it is not.

Therefore, CH-SURF algorithm combines the complementary relationship between the color histogram feature and SURF algorithm.On the basis of the rough positioning, it completes the positioning of target image.

\section{EXPERIMENTAL RESULTS AND ANALYSIS}

In order to test the validity of the CH-SURF algorithm, some experiments are carried out on the VS2008 platform by calling Opencv2.3 Vision Library Function. Experimental source image is the car image of JPEG format,and the target images are one part of source image,they are shown in Fig 3.

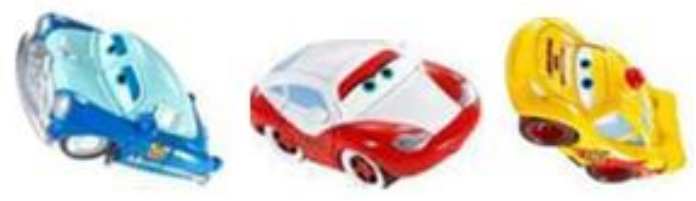

Figure 3. Target images

\section{A. Simulation result}

\section{1) Feature matching results of $\mathrm{CH}-\mathrm{SURF}$}

The feature matching process of CH-SURF algorithm is firstly adopting color histogram method to localize the rough position of target image, secondly using SURF algorithm in the located region to realize accurate positioning. Experimental result is shown in Fig 4.

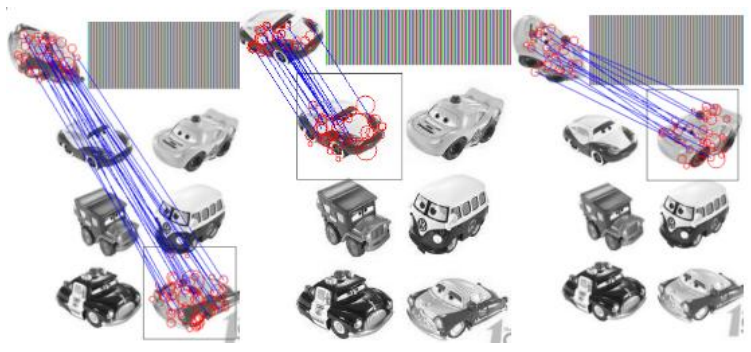

Figure 4. Matching result of $\mathrm{CH}-\mathrm{SURF}$
The black square has drew out the rough location of target image in source image, and the red circles in both the target image and source image, represent the extracted SURF key points, and the matched points are connected by blue lines.

2) Feature matching results of SURF

SURF algorithm is implemented by extracting feature points in the whole source image region,and let them compare with those points extracted by target image. Finally,the one-to-one relationship between feature pairs is connected. Experimental results is shown in Fig 5:

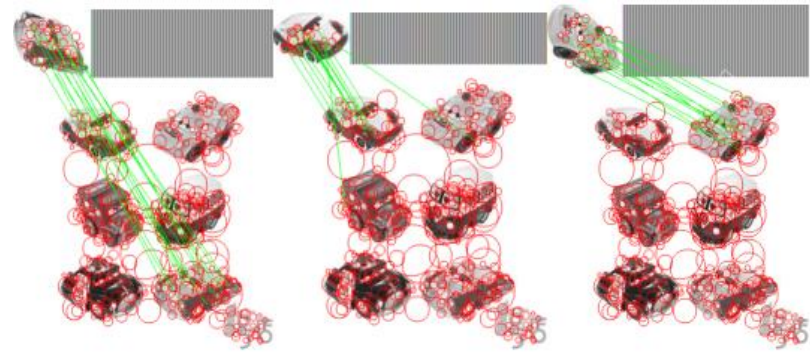

Figure 5. Matching result of SURF

The red circles represent the extracted SURF points in both target image and source image, and the matched points are connected by green lines. But for SURF algorithm,the detected key points are enormous,so it has expensive computation.Moreover SURF algorithm ignores color information, thus it is hard to recognize the image that has similar shape but different color and easy to generate mismatch phenomenon, just as the car02 situation in Fig.5

\section{B. The comparison of CH-SURF and SURF algorithm}

CH-SURF algorithm is based on rough localization of color matching method, so it only needs to detect key points in the rectangular region of source image; while the SURF algorithm has to detect all key points in the whole source image.But,no matter for which algorithms, the detected key points in the target image is the same.Thus we just need to compare CH - SURF algorithm and SURF algorithm about the performance of the source image feature extraction.Here some experiments are carried out and the results are shown in both Table 1 and Table 2.

TABLE I. THE COMPARISON OF THE DETECTED KEY POINTS IN THE SOURCE IMAGE

\begin{tabular}{|c|l|l|l|}
\hline Quantity & Car01 & Car02 & Car03 \\
\hline $\begin{array}{c}\text { The detected } \text { key } \\
\text { points by } \text { CH- } \\
\text { SURF algorithm }\end{array}$ & 54 & 38 & 41 \\
\hline $\begin{array}{c}\text { The detected } \text { key } \\
\text { points by SURF } \\
\text { algorithm }\end{array}$ & 406 & 406 & 406 \\
\hline $\begin{array}{c}\text { Performance } \\
\text { comparison }\end{array}$ & $86.69 \%$ & $\begin{array}{c}\text { decrease } \\
\text { decrease }\end{array}$ \\
\hline
\end{tabular}


In the Table 1,statistical results on a great number of feature descriptors illustrate that, the detected key points by CH-SURF algorithm is obviously less than the SURF algorithm.Take car01 car02 and car03 for example, the number of extracted feature points by CH-SURF algorithm from SURF required 406 reduced to 54、 406 and 38, and its reduction ratio is $86.69 \%, 90.64 \%$ and $89.90 \%$ respectively.

TABLE II. THE COMPARISON OF COMPUTATION TIME IN THE SOURCE IMAGE

\begin{tabular}{|c|l|l|l|}
\hline Quantity & Car01 & Car02 & Car03 \\
\hline $\begin{array}{c}\text { The computation } \\
\text { time of CH- } \\
\text { SURF/ms }\end{array}$ & 25.8196 & 21.7741 & 22.3324 \\
\hline $\begin{array}{c}\text { The computation } \\
\text { time of SURF/ms }\end{array}$ & 148.017 & 147.132 & 146.997 \\
\hline $\begin{array}{c}\text { Performance } \\
\text { comparison }\end{array}$ & $82.56 \%$ & $85.20 \%$ & $\begin{array}{l}\text { decrease } \\
84.81 \%\end{array}$ \\
\hline
\end{tabular}

In the Table 2, the computation time by CH-SURF is also fewer than SURF, feature points extraction time from $148.017 \mathrm{~ms} 、 147.132 \mathrm{~ms}$ and $148.017 \mathrm{~ms}$ respectively reduced to $22.8196 \mathrm{~ms} 、 21.7741 \mathrm{~ms}$ and $21.7741 \mathrm{~ms}$ respectively, and its reduction ratio is $82.56 \%, 85.20 \%$ and $84.81 \%$ respectively. Take car01 for example, statistical results on feature points extraction time in the source image are shown in Fig. 6

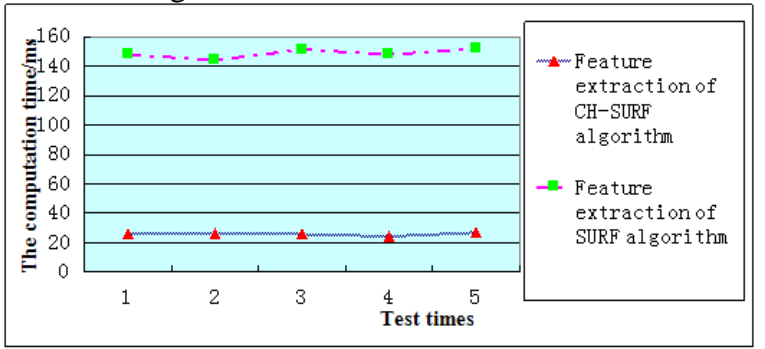

Figure 6. The comparison of computation time of Car01

In the extraction process of the source image feature points, the computation time by the CH-SURF algorithm is obviously fewer than SURF algorithm.In the Fig.6, the CHSURF algorithm computation time ranges from $20 \mathrm{~ms}$ to $25 \mathrm{~ms}$ while the SURF algorithm ranges from $141 \mathrm{~ms}$ to 151 $\mathrm{ms}$.

\section{CONCLUSIONS}

This paper introduced the CH-SURF algorithm which combines the method of color histogram features matching and SURF algorithm together. Meanwhile some experiments are carried out to test the performance of CH-SURF, and results show that the proposed algorithm can make full use of the complementarity relation of two algorithm, and improve insensitive to color information. CH-SURF algorithm is implemented on the based of rough positioning, so the key points extracted reduced by $89 \%$ and its computation time also reduced by $84 \%$ compared with SURF algorithm. Hence experimental results show that the CH-SURF algorithm is effective.

\section{REFERENCES}

[1] Bay H , Tuyteplaars T and van Gool L . SURF: Speeded Up Robust Features [C]. Processings of the European Conference on Computer Vision, 2006:404-417.

[2] Xiujuan Chai, Shiguang Shan, Laiyun Qing, et al: Pose and Illumination Invariant Face Recognition Based on 3D Face Reconstruction[J]. Journal of Software ,2006(3).

[3] Leqing Zhu: Using SURF to recognize palm image. 2010 International Conference on Remote Sensing (ICRS 2010).

[4] Xiaofan Lin, Liwen Lin,Tao Deng :Remote Sensing Image Registration Based on SURF Descriptor[J]. Computer Engineering ,36(12):216-219,2010.

[5] Chenhang Lin , Zhibin Pan, Bin Zou: Color Image Retrieval Algorithm Based on Global and Local Color Features[J]. Microelectronics \& Computer,29(4):104-110,2012.

[6] Hui Huang, Lizhong Lu, Bin Yan, Jian Chen : A New Scale Invariant Feature Detector and Modified SURF Descriptor. 2010 Sixth International Conference on Computation(ICNC 2010).

[7] Lowe D G. Object Recognition from Local Scale-invariant Features . International Conference on Computer Vision. Corfu:1999:1150-1157

[8] Lowe D G: Distinctive Image Features from Scale-invariant Keypoints . International Journal of Computer Vision. 60(2):91110,2004.

[9] CHEN Bing, Yigong Zhao, Xin Li: Scene matching based on speeded up robust features[J]. Systems Engineering and Electronics, 31 (11):2714-2719,2009.

[10] Qi Liu, Mingyi He: Image Stitching Based on SURF Feature Matching[J]. Measurement \& Control Technology. 29(10):2732,2010. 